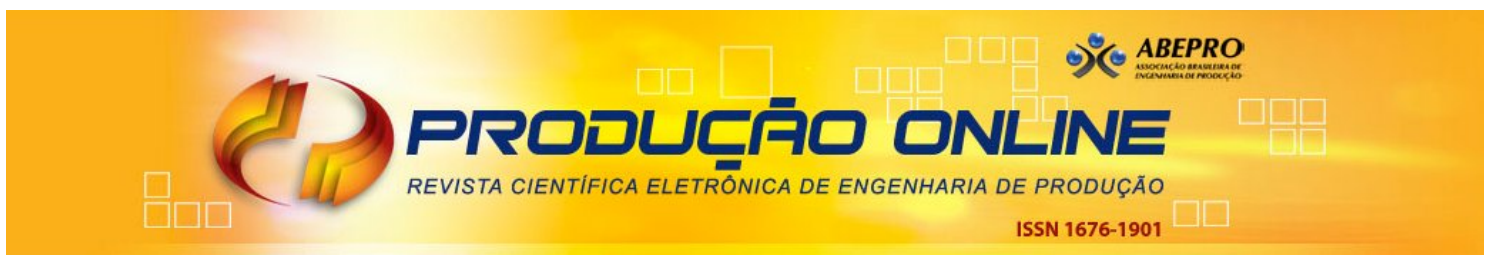

\title{
TPM E MANUTENÇÃO AUTÔNOMA: ESTUDO DE CASO EM UMA EMPRESA DA INDÚSTRIA METAL-MECÂNICA
}

\author{
TPM AND AUTONOMOUS MAINTENANCE: CASE STUDY IN A METAL \\ MECHANIC COMPANY
}

\author{
Norberto Carvalho Biehl* E-mail: norbertobiehl@hotmail.com \\ Miguel Afonso Sellitto* E-mail: sellitto@unisinos.br \\ *Universidade do Vale do Rio dos Sinos (UNISINOS), São Leopoldo, RS
}

\begin{abstract}
Resumo: O objetivo deste artigo foi descrever um caso de aplicação de manutenção autônoma em uma área-piloto de uma empresa da indústria metal-mecânica. O método de pesquisa foi o estudo de caso. A TPM - Total Productive Maintenance, Manutenção Produtiva Total, é uma tecnologia gerencial que pode auxiliar as empresas na redução de ocorrência de falhas e quebras de máquinas. Um dos mais importantes aspectos da TPM é a manutenção autônoma. Um programa de doze pontos foi estruturado e adotado como guia de implantação da manutenção autônoma. O programa iniciou por uma área-piloto, incluindo três centros de usinagem. Os principais resultados do programapiloto foram o aumento em mais de $700 \%$ no tempo médio entre falhas, a redução de mais de $40 \%$ no tempo médio até o reparo, o consequente aumento de mais de cinco pontos percentuais na disponibilidade das três máquinas e a redução de quase $60 \%$ no custo de materiais de manutenção. A principal conclusão foi que a TPM, e em particular a manutenção autônoma, pode aumentar a eficiência da manutenção como fator estratégico, aumentando a capacidade de competição da manufatura em empresas industriais.
\end{abstract}

Palavras-chave: TPM. Redução de custo. Manutenção autônoma.

\begin{abstract}
The purpose of this article was to describe a case of autonomous maintenance implementation in a pilot area of a metal mechanical industry company. The TPM - Total Productive Maintenance is a managerial technology that can help companies to reduce failures and breakages of machines. One of the most important issues of TPM is autonomous maintenance. The method of research was the case study. A program of twelve points was structured and adopted as stand-alone maintenance deployment guide. The program began in a pilot area, including three machining centers. The main results of the pilot program were: increasing by more than $700 \%$ the Mean Time between Failure, reducing by more than $40 \%$ in the Mean Time to Repair, as well as the consequent increase of more than five percentage points on the availability of the three machines and almost $60 \%$ reduction in the cost of maintenance materials. The main conclusion was that the TPM, and in particular the autonomous maintenance, can increase the efficiency of maintenance as strategic factor, which enhances the competitiveness of manufacturing in industrial companies.
\end{abstract}

Keywords: TPM. Cost-cutting. Autonomous maintenance.

\section{INTRODUÇÃO}

As empresas industriais têm buscado medir a eficiência de seus sistemas produtivos, identificando e eliminando perdas, de modo a manter a competitividade 
por meio de seus sistemas produtivos (RAPOSO, 2011). Em decorrência do acirramento da competitividade, empresas e indústrias têm procurado priorizar ganhos de produtividade, eficiência e qualidade nas suas operações. Neste contexto, sistemas de produção com alta confiabilidade têm sido apontados como fator crítico para a construção de vantagem competitiva na indústria (SINGH et al., 2014). Para tanto, a manufatura deve ser suportada por estratégias de manutenção que sejam eficientes e efetivas (BAMBER et al., 1999). Com isto, a manutenção tem se tornado fator relevante na estratégia de competição de empresas industriais (AHUJA, 2012).

Um modo de aumentar o desempenho da manutenção é formular e implementar uma estratégia baseada em manutenção produtiva total (TPM ou Total Productive Maintenance) (SINGH et al., 2014). A atividades de TPM se concentram em melhorias e na otimização do uso do ativo industrial, com custos de produção baixos, preços competitivos, e equipe de produção capacitada, motivada e focada em resultados (MONTEIRO et al., 2012). A TPM aborda o ciclo de vida de equipamentos, minimizando avarias, defeitos de produção e acidentes com danos pessoais e patrimoniais (ALCARAZ, 2011). Junto com outros métodos associados à manufatura enxuta, a TPM pode contribuir para o sucesso e sobrevivência de empresas e indústrias (PACHECO et al., 2012; MUTIAH et al., 2008).

Um dos aspectos da TPM é a manutenção autônoma, que inclui técnicas que permitem que os operadores conservem o equipamento no melhor estado possível, com participação apenas eventual do setor de manutenção e de construção de máquinas, que dão apenas embasamento e sustentação aos operadores nas atividades de manutenção de suas próprias máquinas (GOMES et al., 2012). A manutenção autônoma envolve operadores na manutenção de suas próprias máquinas, com suporte do departamento central de manutenção (TAKAHASHI e OSADA, 2000), o que aumenta o nível de motivação e comprometimento com o estado do equipamento de equipes de operação (GRAISA e AL-HABAIBEH, 2011).

A implantação do TPM tem resultado em aumentos de eficiência na utilização da capacidade instalada em indústrias japonesas que têm oscilado entre 60 e 90\% (TONDATO, 2004). Uma decorrência deste aumento de eficiência é a redução na necessidade de novos investimentos de capital na planta, pois é possível produzir mais com o mesmo ativo (DOGRA et al., 2011). Chan et al. (2005) relataram um 
caso em que a TPM trouxe ganho de $83 \%$ de produtividade na indústria eletrônica. Tsang e Chan (2000) relatam um caso na indústria metal-mecânica em que o número de quebras em máquinas CNC caiu mais de $50 \%$ em dois anos. Sivakumar (2014) relata um caso de de redução de 48\% de defeitos em encapsulamentos de chips. Os autores destacaram que resultados de longo prazo surgiram a partir de aprendizado em projetos-piloto. Ljungberg (1998) apresenta casos em que, devido a programas estruturados de implantação de TPM, houve sensível redução de refugos e retrabalhos, ao mesmo tempo em que houve redução no número de quebras de máquinas e interrupção inesperada da produção. Em síntese, a implantação da TPM tem relação positiva e significante com redução de custo, aumento de qualidade e atendimento de prazos de entrega (MCKONE et al., 2001).

A empresa Alfa, assim denominada por motivos de sigilo empresarial, vem se deparando com problemas de qualidade em produtos e com quebras de máquinas, gerando altos custos devidos à perda de produtividade. Após visitas e estudos, a empresa decidiu investir na implantação do programa TPM como forma de aprimorar seus processos produtivos, iniciando pela manutenção autônoma. O objetivo deste artigo é descrever um caso de aplicação de manutenção autônoma em uma áreapiloto de uma empresa da indústria metal-mecânica. A questão de pesquisa foi: como aplicar a manutenção autônoma em uma empresa da indústria metalmecânica? O método de pesquisa foi o estudo de caso descritivo. Os objetivos secundários foram: descrever o cenário em que a aplicação ocorreu; descrever procedimentos e resultados; e analisar os benefícios obtidos, comparando resultados antes e após a implantação do programa. Este artigo limita-se a uma área piloto da empresa, incluindo três centros de usinagem.

\section{TPM E MANUTENÇÃO AUTÔNOMA}

Programas formais de Melhoria Contínua (MC) apareceram pela primeira vez no Japão, na segunda metade do século XX (JHA et al., 1996). Depois da segunda guerra mundial, as indústrias japonesas determinaram que para competir com o sucesso mundial na fabricação, era necessário desenvolver qualidade em seus produtos. Dentro deste contexto, os japoneses importaram técnicas de 
gerenciamento e fabricação dos Estados Unidos adaptando-as para as suas circunstâncias (BHUIYAN e BAGHEL, 2005).

Ocorre, a partir de então, expressiva disseminação de programas de MC. Isso se deve em parte à modernização das práticas gerenciais, em que as relações humanas no trabalho passam a ser incorporadas à rotina das empresas. Há ainda o acirramento da concorrência e, por conseguinte, da necessidade de aumento de competitividade, gerando pressão por desempenhos em produtividade, melhor qualidade dos produtos e serviços, flexibilidade de volume e de produção, rapidez no atendimento aos clientes e inovações dos produtos (JHA et al., 1996; BHUIYAN e BAGHEL, 2005).

No século XXI, têm-se adotado técnicas de inovação e aprendizado organizacional como parte da estratégia competitiva, que teve como suporte os programas de melhoria contínua baseado na abordagem japonesa do Kaizen. A organização e operacionalização das empresas, para essa nova estratégia competitiva, vieram com novos programas, como a TPM, que focou a agilidade e flexibilidade de produção (BOER e GERTSEN, 2003).

Comparados com as décadas passadas, os desafios atuais enfrentados pelas empresas são mais complexos, exigem maiores competências técnicas e mais habilidades gerenciais. Também exigem a utilização mais eficiente de técnicas e ferramentas destinadas à identificação e solução de problemas de produção e de manutenção (BESSANT et al., 1994; BESSANT e CAFFYN, 1997; BESSANT, 1999; BESSANT et al., 2001). Muitas destas técnicas têm como objetivo fazer previsões sobre os momentos mais prováveis em que uma falha pode ocorrer e deste modo antecipar-se à falha por meio de uma decisão de intervenção focada (SELLITTO et al., 2002). Tal técnica é especialmente útil quando existe um ou poucos equipamentos, os gargalos, cujas falhas causam danos muito mais consideráveis na produção do que outras falhas. Neste caso, a aplicação da técnica deve se concentrar nos gargalos (SELLITTO, 2002).

A TPM é um método para gerir atividades industriais e foi divulgado principalmente pelos estudos publicados por Seiichi Nakajima, nos anos 1980. Seus objetivos principais são melhorar a eficácia e o tempo de vida útil dos equipamentos, eliminando desperdícios no processo de produção ( $\mathrm{KOCH}, 2007)$. Oficialmente, a sigla TPM surgiu em 1971, cunhada pela JIPE (Japan Institute of Plant Engineering, 
Instituto Japonês de Engenheiros de Fábrica). A ênfase inicial estava na introdução da metodologia apenas nas plantas fabris, mas depois a mesma foi estendida aos demais setores da atividade empresarial (NAKAMURA, 2007). No início, a TPM estava restrita ao setor de produção e tinha como finalidade: (i) maximizar a eficiência do equipamento (overall equipment efficiency); ii) estabelecer um sistema total de manutenção, projetado para a vida inteira do equipamento; e (iii) operar em todos os setores envolvidos com equipamentos, incluindo planejamento, operação e manutenção (SUZUKI, 1994).

A TPM tem como base a participação de todos os membros da empresa, desde a alta gerência até os colaboradores de campo e é realizada principalmente por meio de atividades em pequenos grupos (SUZUKI, 1994). Yoshicazem (2002) cita que a TPM surgiu no Japão em 1971, visando ao aperfeiçoamento de técnicas existentes de manutenção preventiva e engenharia de confiabilidade, visando à falha zero nos processos, quebra zero dos equipamentos e perda zero nos processos.

A TPM dirigiu sua atenção para a redução de custos do equipamento no seu ciclo de vida, combinando manutenção preventiva com melhorias sustentáveis e projeto de manutenção preventiva. A TPM significa uma manutenção autônoma da produção que tenta aperfeiçoar a habilidade do operador e o conhecimento do seu próprio equipamento para aumentar, ao máximo, a sua eficiência de operação (AHMED et al., 2005).

A principal evolução da TPM ocorreu no final dos anos 1980, com a introdução da TPM empresarial. Seus objetivos são: (i) criar um sistema corporativo que maximiza a eficiência do sistema de produção (overall efficiency improvement); (ii) criar sistemas para impedir a ocorrência de todas as perdas na linha; e (iii) estar focado no produto acabado (SHIROSE e BODEK, 1992). Segundo Nakazato (2000), usualmente as empresas que implantam a TPM industrial alcançam bons resultados locais. À medida que as atividades da TPM se estendem a toda a empresa, os resultados também se estendem, transformando-se em ganhos objetivos de competitividade, tais como custo de produção menor, prazos de entrega menores e qualidade de conformação do produto maior. 


\subsection{Objetivos da TPM}

Os objetivos da TPM foram definidos pelo JIPM (Japan Institute of Plant Maintenance - Instituto Japonês de Manutenção Industrial): (i) Maximizar a eficiência dos sistemas produtivos; (ii) Minimizar perdas, estabelecendo metas orientadas a zero acidentes, zero perdas e zero defeitos, ao longo do ciclo de vida dos elementos produtivos; (iii) Envolver todos os departamentos na implantação, incluindo novos produtos, vendas e administração; (iv) Envolver todos os funcionários desde a alta administração até os operadores de chão de fábrica; e (v) Agir por atividades de pequenos grupos (APG) (SUZUKI, 1994).

Para maximizar a eficiência da planta deve ser eliminado tudo o que reduz a eficiência, ou sejam, as perdas. A ideia central do programa é a completa eliminação das seis principais perdas nas máquinas: quebras, tempos de troca de ferramentas e regulagem (setup), tempos de esperas, velocidade reduzida, defeitos na produção (refugos e retrabalhos), e queda de rendimento no início de turno (NAKAZATO, 1999). Portanto, o fundamento da TPM é que o rendimento operacional máximo seja alcançado pela eliminação da totalidade das perdas. Na prática, tal resultado é de difícil obtenção, porém um índice de rendimento operacional próximo a 85\% é perfeitamente alcançável na maior parte das empresas industriais (NAKAJIMA, 1989).

Nakajima (1989) definiu seis grandes perdas em equipamentos, que podem influenciar diretamente na produtividade fabril. Estas seis grandes perdas são descritas no Quadro 1 e foram sintetizadas por Tondato (2004), que usou e referenciou Salvendy (1992), Wireman (2004), Shirose (1992), e Kardec e Nascif (1998). 
Quadro 1 - As seis grandes perdas de produtividade combatidas pela TPM

\begin{tabular}{|c|c|}
\hline Tipo de perda & Descrição \\
\hline Perdas por quebra & $\begin{array}{l}\text { Falha ou quebra é a perda de uma função especifica de um objeto, } \\
\text { tal como uma peça ou componente, que ocorra de forma inesperada } \\
\text { e acarrete parada de equipamento. }\end{array}$ \\
\hline $\begin{array}{l}\text { Perdas devido a } \\
\text { ajustes e setup }\end{array}$ & $\begin{array}{l}\text { São perdas de tempo observadas em equipamentos utilizados para } \\
\text { vários produtos, principalmente para o set-up e o ajuste de qualidade. }\end{array}$ \\
\hline $\begin{array}{l}\text { Perdas por } \\
\text { pequenas paradas } \\
\text { e trabalho lento ou } \\
\text { em vazio }\end{array}$ & $\begin{array}{l}\text { São interrupçães de curto prazo que exigem imediata intervenção } \\
\text { dos operadores para normalizar a produção. Exemplos: trabalho em } \\
\text { vazio pelo entupimento do sistema de alimentação; detecção de } \\
\text { produtos não conforme por sensores e consequentemente parada da } \\
\text { linha de produção e sobrecarga ocasionando o desligamento. }\end{array}$ \\
\hline $\begin{array}{l}\text { Perdas por } \\
\text { redução da } \\
\text { velocidade }\end{array}$ & $\begin{array}{l}\text { Perdas devidas à redução da velocidade são definidas como a } \\
\text { diferença entre a capacidade nominal do equipamento e a } \\
\text { capacidade no qual o mesmo está operando. Os dois maiores fatores } \\
\text { de impacto na capacidade do equipamento são a velocidade e o } \\
\text { volume de produção. }\end{array}$ \\
\hline $\begin{array}{l}\text { Perdas por } \\
\text { problemas de } \\
\text { qualidade }\end{array}$ & $\begin{array}{l}\text { Estas perdas são relacionadas com defeitos de qualidade, } \\
\text { retrabalhos ou reparos e podem ocorrer de forma ocasional e } \\
\text { habitual. Os ocasionais ou especiais são causados por } \\
\text { funcionamento inadequado, ou pelo aparecimento repentino de um } \\
\text { problema. Os habituais ou comuns são causados pela operação do } \\
\text { equipamento com algum grau de deterioração que começou a ser } \\
\text { aceito como operação normal; ou seja, causados geralmente por } \\
\text { problemas antigos diretamente relacionados ao equipamento, que } \\
\text { nunca foram levados em conta. }\end{array}$ \\
\hline $\begin{array}{l}\text { Perdas de } \\
\text { rendimento ou na } \\
\text { partida }\end{array}$ & $\begin{array}{l}\text { Essas perdas ocorrem sempre que um processo precisar ser } \\
\text { interrompido e depois, reiniciado, tendo a ver com a produção de um } \\
\text { item inaceitável quando o equipamento, por exemplo, atinge um certo } \\
\text { parâmetro limítrofe de processo, tal como temperatura, velocidade ou } \\
\text { pressão. }\end{array}$ \\
\hline
\end{tabular}

\subsection{Os Oito Pilares da TPM}

Para a eliminação das seis grandes perdas, implementam-se oito tipos de atividades: os oito pilares de sustentação da TPM (NAKAZATO, 1999). A operacionalização da TPM está organizada em uma estrutura de abordagem baseada nos oito pilares (LAZIM et al., 2008; AHUJA e KHAMBA, 2008). Os pilares devem ser desenvolvidos em equipes, tendo como foco as dimensões PQCDSM (produtividade, qualidade, custos, atendimento ao cliente, segurança e moral). A Figura 1 apresenta os pilares (SINGH et al., 2013). 
Figura 1 - Os pilares de sustentação do programa TPM

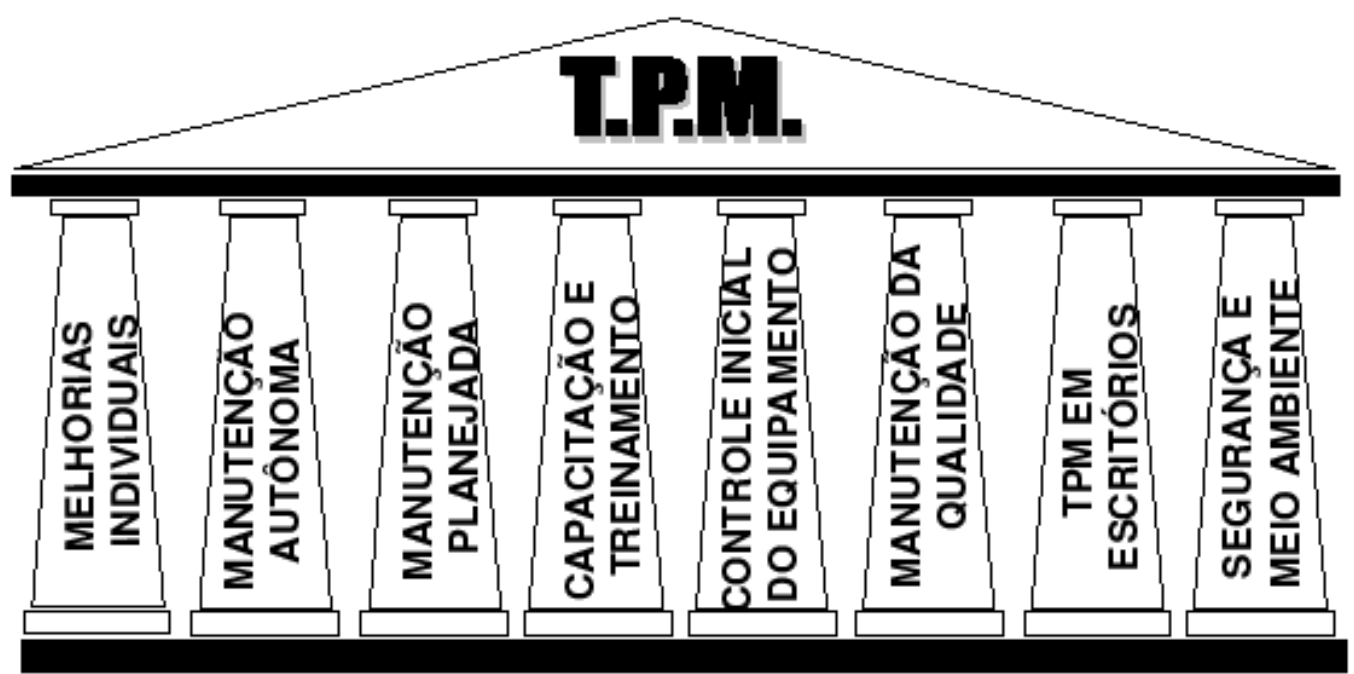

Fonte: SINGH et al. (2013)

Os pilares são (SOUZA, 2001; TONDATO, 2004):

- Pilar de Melhorias Específicas (ou individuais): busca auxiliar no entendimento das maiores perdas de cada área ou equipamento, além da implantação das melhorias a fim de reduzi-las (SOUZA, 2001). As melhorias individuais são atividades que buscam otimizar a eficiência dos equipamentos, pela eliminação sistêmica das perdas. Elas são necessárias devido à baixa eficiência das ações de melhoria contínua. As melhorias decorrentes do dia a dia podem não ocorrer de forma desejada devido a uma série de fatores. Por isso, o pilar de melhorias individuais administra este tipo de tarefa (SUZUKI, 1993);

- Pilar de Manutenção Autônoma: envolve e ensina os operadores a trabalhar em equipe e de modo flexível, a conhecer e operar melhor os equipamentos, descobrir deficiências e identificar pontos a melhorar de forma contínua (RAJPUT e JAYASWAL, 2012). A manutenção autônoma usa os operadores na manutenção e preservação dos equipamentos, em atividades como limpeza, lubrificação, inspeção, e pequenos reparos (TONDATO, 2004);

- Pilar de Manutenção Planejada: tem como principal objetivo aumentar a eficiência do equipamento buscando a quebra zero e evitando variabilidade excessiva na produção (SOUZA, 2001). Para isso, a equipe de manutenção deve desenvolver atividades preventivas e de controle de máquinas. Pensando nisso, é de se esperar que as empresas que buscam um processo 
de qualidade total, ou estabilizado, disponibilizem tempo da produção para paradas de manutenção preventiva. Ocorre que, à medida que se avança ao encontro da qualidade total e da produção enxuta, percebe-se que, ainda que o tempo de interrupção para manutenção venha a prevenir um tempo ainda maior de parada devido a uma quebra advinda de uma falta de atuação preventiva, ele reduz a utilização da máquina, e pode ser visto como perda por tempo de espera, sendo reduzido (LEÃO, 2009);

- Pilar de Educação (capacitação) e Treinamento: visa possibilitar o aumento do conhecimento, desenvolvimento de habilidades e mudanças comportamentais. Suas principais ferramentas são a matriz de habilidades e as lições ponto-a-ponto (SOUZA, 2001);

- Pilar de Controle Inicial: tem como objetivo garantir bom desempenho inicial do equipamento adquirido por uma abordagem sistemática de especificação e realimentação de informações de projeto a fornecedores (SOUZA, 2001);

- Pilar de Manutenção da Qualidade: também conhecido como gerenciamento MQP (máquinas, qualidade, pessoas), é, por vezes visto, erroneamente, como meras melhorias em equipamentos. No entanto, o gerenciamento MQP busca atingir e assegurar a qualidade total identificando e controlando as relações entre a qualidade dos produtos e a deterioração tanto das condições de processo, como de partes dos equipamentos. Este procedimento deve ser realizado durante toda a vida útil do equipamento (TSUCHIYA,1992);

- Pilar de Melhoria nos Processos Administrativos: tem como principal objetivo aumentar a velocidade e a qualidade das informações que passam pelas áreas, além de eliminar o processamento desnecessário de informações (SOUZA, 2001); e

- Pilar de Saúde, Segurança e Meio Ambiente: busca a prevenção de acidentes, além de preservar o meio ambiente das influências negativas que os equipamentos de operação possam trazer (SOUZA, 2001).

\subsection{Manutenção Autônoma}

Dentre os diversos aspectos do contexto maior da TPM, para este artigo interessa mais enfocar na manutenção autônoma (MA). 
A MA enfoca principalmente a eliminação das perdas geradas pela má utilização de equipamentos e recursos humanos, eliminando não-conformidades e desenvolvendo, por meio dos operadores, pequenas e contínuas melhorias locais (PETTER et al., 2011). Segundo Wyrebski (1997), a MA visa à maximização da utilização de equipamentos e processos. Sua prática continuada pode desenvolver e fazer aflorar conhecimentos e reeducar operadores, exigindo postura pró-ativa, prevenção de problemas, e senso de melhoria contínua, o que garante o aumento da confiabilidade e da disponibilidade nos equipamentos sem novos investimentos em maquinaria. Para Tavares (1999), a MA envolve os operadores nas atividades de manutenção, lubrificação, inspeções visuais e limpeza, tendo como base à prática continuada e sistemática do Método $5 \mathrm{~S}$.

Segundo Nakajima (1989), Tavares (1999) e Venkatesh (2003), a implantação da manutenção autônoma é realizada em sete etapas:

- Etapa 1: limpeza inicial (combate à sujeira);

- Etapa 2: medida de combate as fontes geradoras de problemas e locais de difícil acesso (eliminação das sujeiras em sua origem);

- Etapa 3: elaboração de padrões de inspeção, lubrificação, limpeza e reaperto (a busca de um estado ideal no local de trabalho);

- Etapa 4: inspeção geral (desenvolver operadores capazes de compreender as funções básicas do equipamento, identificar defeitos e efetuar reparos);

- Etapa 5: inspeção autônoma (verificação dos processos das atividades de inspeção, limpeza, lubrificação e reaperto);

- Etapa 6: organização e ordem (organização e cuidado com ambiente de trabalho);

- Etapa 7: consolidação da manutenção autônoma (verificações das etapas anteriores e execução de melhorias para incremento da eficiência do equipamento).

A autonomia concedida aos operadores em MA é baseada nas chamadas atividades JK (Jishu Kanri - controle autônomo). Tais práticas consolidam a ideia de que cada operador executa e controla seu próprio trabalho com autonomia, sem supervisão. Em síntese, a autonomia na manutenção significa que cada operador pode e deve cuidar de seu próprio equipamento (YAMAGUCHI, 2005). Yamaguchi 
(2005) complementa que a MA pode ser entendida como um processo de capacitação de operadores, com o objetivo de torná-los aptos a desenvolver mudanças que possam garantir altos níveis de produtividade fabril e de disponibilidade dos equipamentos. A MA pode ser compreendida como uma mudança de conceito: antes era "eu fabrico, você conserta"; agora é "do meu equipamento cuido eu" (PETTER et al., 2011).

A MA está entre os métodos mais eficazes para a transformação de uma fábrica em uma operação focada nos equipamentos, conferindo a estes características de confiabilidade, disponibilidade, produtividade, e segurança operacional. Tais elementos podem ser importantes para o desenvolvimento de características de competitividade, tais como alta qualidade, prazos reduzidos, e baixos custos de produção (TAKAHASHI, 1993).

\section{A PESQUISA}

O objetivo deste artigo é descrever um caso de aplicação de manutenção autônoma em uma área-piloto de uma empresa da indústria metal-mecânica. O método de pesquisa foi o estudo de caso único, realizado na empresa Alfa. Como fontes de evidências, foram empregados documentos internos da empresa, entrevistas com gestores e com operadores e a participação do pesquisador. As principais técnicas de pesquisa foram a entrevista semi-estruturada e a observação participante.

A empresa Alfa é uma das três maiores fabricantes de armamentos do mundo, com mais de 70 anos de história e mais de 4.500 funcionários. A empresa produz para os mercados de forças militares, civis (defesa e caça esportiva) e policiais, diversos modelos de armas. A empresa exporta para diversos países e já recebeu prêmios internacionais de qualidade emitidos por entidades do setor de armamentos. A empresa investe em design e tecnologia, sendo pioneira na utilização de polímeros de titânio na fabricação de armamentos. O projeto de implantação da TPM fez parte do esforço feito pela empresa para alcançar suas metas de qualidade em produtos e eficiência em processos produtivos.

O método de trabalho para a elaboração da pesquisa foi:

- Revisão bibliográfica sobre TPM e manutenção autônoma; 
- Estabelecimento do método de implantação, baseado na revisão da obra de Nakajima (1988);

- Descrição das atividades conduzidas em campo e relato dos resultados numéricos até agora obtidos (sessão 3.1);

- Análise dos resultados até agora obtidos e projeção de continuidade (capítulo $4)$.

O método de implantação da manutenção autônoma foi:

(i) comprometimento da alta administração;

(ii) divulgação dos procedimentos a serem adotados;

(iii) definição do comitê responsável pelo projeto;

(iv) determinação das políticas e metas básicas;

(v) definição do plano diretor;

(vi) início propriamente dito da implantação;

(vii) instrução dos operadores;

(viii) definição de procedimentos pelos grupos de trabalho;

(ix) escolha de equipamentos-piloto para medição de eficácia;

(x) desenvolvimento da manutenção autônoma;

(xi) medição de resultados;

(xii) realização de auditoria e retorno ao início.

\subsection{Descrição das atividades}

No início de 2013, a empresa decidiu redefinir alguns conceitos centrais na função produção, inclusive com mudanças na diretoria industrial. Esta diretoria manifestou a necessidade de implantação do programa TPM, com o objetivo de auferir os possíveis benefícios que este programa pode oferecer.

Por diagnóstico preliminar, foram detectados os seguintes problemas: grande número de quebra de máquinas, falta de registros e estatísticas destas quebras, má condição das máquinas, falta de instrução de operadores. Após o diagnóstico, a diretoria promoveu uma reunião com os gerentes de unidade declarando oficialmente a implantação do programa TPM nos parques fabris. Fez parte da atividade a divulgação dos objetivos a serem adotados. 
Com esta medida, implantou-se o passo (i).

Cada gerente ficou responsável pela coordenação de implantação em sua unidade. O programa teve início em 2013, com a definição de uma equipe composta por profissionais de diversas áreas (PCP, manutenção, engenharia e produção), que seriam os líderes, responsáveis pelos primeiros passos do programa. Com o objetivo de capacitar a liderança escolhida, foram promovidas atividades de treinamento, com consultores externos associados a lideranças internas, com duração de 30 horas, a fim de abordar os temas necessários para o gerenciamento e planejamento das atividades relativas à implantação do programa TPM.

Com esta medida, implantaram-se os passos (ii) e (iii). O Quadro 2 apresenta os temas constantes da atividade.

Quadro 2 - Treinamento dos líderes

$\checkmark$ Definição, objetivos e princípios do TPM

$\checkmark$ Oito pilares do programa TPM

$\checkmark$ Metodologia de implantação

$\checkmark$ Classificação das perdas

$\checkmark$ Ferramentas de trabalho (5S, 5W1H, Diagrama de Ishikawa, Diagrama de Pareto, entre outros)

A implantação do programa iniciou-se em janeiro de 2013. Seus objetivos gerais foram: redução no número e na gravidade das avarias em máquinas, redução da quantidade de intervenções emergenciais em máquinas, redução no tempo total gasto com máquinas paradas, redução dos custos totais de manutenção e mudança de cultura das equipes quanto a responsabilidades e comprometimentos com a situação e o estado dos equipamentos de produção.

Foi eleita uma área-piloto contendo três centros de usinagem para iniciar as atividades e ganhar conhecimento acerca do processo. Para esta área, foram determinados como objetivos específicos: redução do tempo até o reparo das três máquinas, aumento do intervalo entre falhas das três máquinas, e redução de custo total de manutenção das três máquinas. Foi definido que, após esta introdução, e segundo os resultados iniciais, será projetada a ampliação do programa para outras áreas da empresa.

Com esta medida, implantaram-se os passos (iv), (v) e (vi).

Após a preparação inicial, os líderes da área escolhida, apoiados por consultores externos, atuaram como multiplicadores, responsáveis pelos 
treinamentos dos supervisores e colaboradores da produção. Foram abordados nesta atividade os principais conceitos da TPM e agregadas atividades de conscientização acerca de sua importância para a empresa. Este treinamento teve duração de doze horas.

Com esta medida, implantou-se o passo (vii).

Neste treinamento, foi apresentado e discutido o check-list para preenchimento diário pelos operadores. O check-list consiste em uma planilha de inspeção diária do equipamento, que deve ser visualizada e preenchida pelo operador, com o objetivo de identificar futuros problemas e paradas inesperadas do equipamento. Todas as irregularidades apontadas no check-list devem ser sanadas imediatamente. Cabe ao operador decidir se a responsabilidade pelo saneamento da situação cabe aos operadores ou à manutenção central. No início do período de implantação, esperava-se que mais atividades corretivas fossem destinadas à manutenção central. À medida que o plano evoluísse, esperava-se que aumentasse a parcela de atendimentos destinados à equipe de operadores. Tal fato realmente ocorreu: ao fim de três meses, mais da metade das irregularidades apontadas pela inspeção diária foram sanadas pela própria equipe de operadores. As ações de melhorias apontadas pela inspeção ou sugeridas pela manutenção central também têm passado paulatinamente para equipe de operadores.

A Figura 2 apresenta o check-list.

Com as equipes treinadas e a área piloto definida, foi executado um treinamento prático na fábrica para que os operadores pudessem ter a oportunidade de testar e interpretar as atividades do check-list e os pontos chaves de verificação dos equipamentos. Os pontos chave de verificação dos equipamentos foram descritos em uma instrução de trabalho (IT) que, a partir de então, passou a ficar anexa às máquinas para ser consultada permanentemente pelo operador.

Com esta medida, implantou-se o passo (viii).

As máquinas escolhidas para o plano-piloto foram os centros de usinagem TM 16488, TM 20500 e TM 18375. Com os check-lists e as instruções de trabalho instaladas nos postos de trabalho, executaram-se as primeiras atividades de limpeza e verificação de máquinas, conjuntamente entre supervisor, líderes da área, a equipe de gestão da TPM, a manutenção central e todos os operadores a fim de promover o evento e dar credibilidade à implantação do programa. Após esta 
primeira atividade, os operadores iniciaram as verificações corriqueiramente e os apontamentos dos check list diariamente. No decorrer deste processo surgiram algumas dúvidas quanto ao preenchimento do documento, que foram sendo sanadas pela equipe da TPM, muitas vezes com apoio da equipe do setor de manutenção central.

Figura 2 - Verificação operacional, o check-list

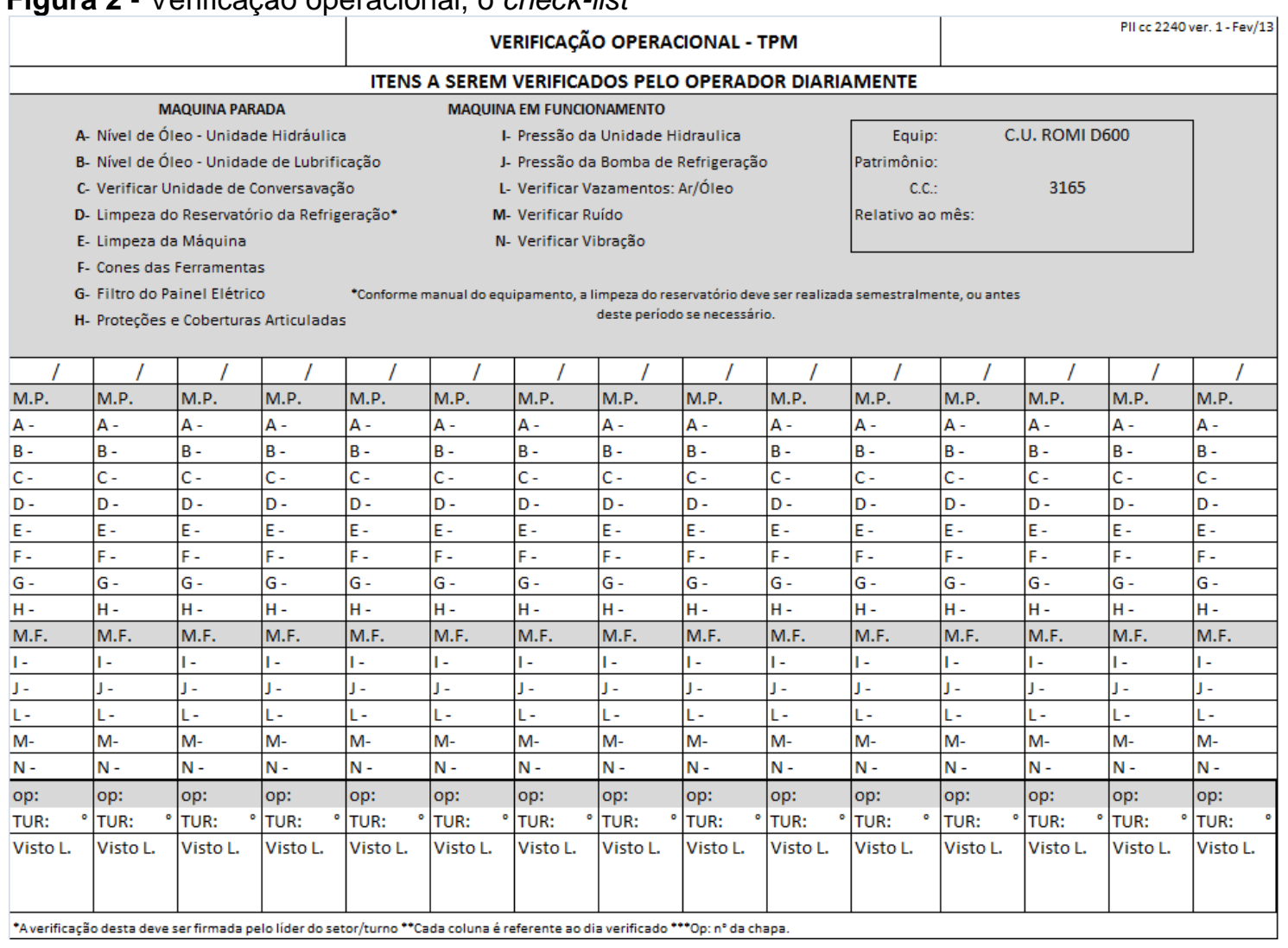

Com esta medida, implantaram-se os passos (ix) e (x).

Os resultados obtidos na implantação da manutenção autônoma como início do processo de TPM são referentes ao plano-piloto, o qual ainda está em fase de implantação. Os dados foram coletados em um período de sessenta dias antes do programa e cento e vinte dias após o início do mesmo. Os resultados foram analisados por meio de medições do MTBF (Mean Time Between Failures, tempo médio entre falhas), do MTTR (Mean Time to Repair, tempo médio de reparo) e do custo de manutenção. Para o cálculo de MTBF e MTTR, são anotados os intervalos entre as falhas e a duração do estado de falha durante um dado intervalo de tempo (por exemplo, três meses). Os tempos médios são obtidos dividindo estes intervalos 
pelo número de falhas. Eventualmente, também pode-se chegar a estes valores por modelagem matemática de distribuições de probabilidade que descrevam os tempos e pelo cálculo do valor esperado destas distribuições (neste artigo usou-se o primeiro método). Tais indicadores permitem o cálculo da disponibilidade de máquina, formando um conjunto de três indicadores que são considerados relevantes para o gerenciamento da manutenção industrial (SELLITTO, 2005).

Com esta medida, implantou-se o passo (xi).

\section{AVALIAÇÃO DE RESULTADOS E DISCUSSÃO}

Para avaliar os resultados até agora obtidos, usaram-se dados comparados de MTBF, MTTR e custos de manutenção. Os dados anteriores foram colhidos entre novembro e dezembro de 2012. Os dados posteriores foram colhidos entre março e agosto de 2013.

A Tabela 1 apresenta dados comparados entre as máquinas escolhidas como piloto para o tempo médio entre as falhas, disposto em horas.

Tabela 1 - MTBF antes e após a implantação da manutenção autônoma

\begin{tabular}{ccccc}
\hline & \multicolumn{3}{c}{ MTBF } \\
\hline \multirow{2}{*}{ Equipamentos } & \multicolumn{3}{c}{ Antes da implantação } & \multicolumn{2}{c}{ Após a implantação } \\
\cline { 2 - 5 } & \multicolumn{1}{c}{ Horas } & Dias & Horas & Dias \\
\cline { 2 - 5 } TM 16488 & $46: 33: 55$ & 1,9 & $314: 35: 04$ & 13 \\
TM 20500 & $58: 16: 39$ & 2,4 & $395: 68: 17$ & 16,5 \\
TM 18375 & $63: 43: 57$ & 2,6 & $505: 22: 43$ & 21 \\
Média & & 2,3 & & 16,8 \\
\hline
\end{tabular}

Antes do início das atividades, as máquinas apresentavam um tempo médio entre falhas expressivamente curto. O período de intervenção entre as manutenções foi em média de dois dias no equipamento TM 16488, e nos outros dois equipamentos, TM 20500 e TM 18375, em menos de três dias. A média das médias ficou em 2,3 dias. Após as atividades, a média entre os tempos médios subiu para 16,8 dias, com um expressivo salto de mais de sete vezes. Das ordens de correção executadas pela manutenção nos dois meses monitorados, constatou-se que cerca de $80 \%$ são de falhas mecânicas e o restante de falhas eletroeletrônicas, 
ocasionadas por desgastes de peças, mau uso ou falta de atenção da parte de preparação e operação dos equipamentos.

A Tabela 2 apresenta dados comparados entre as máquinas escolhidas como piloto para o tempo médio até o reparo, em horas e em minutos.

Tabela 2 - MTTR antes e após a implantação da manutenção autônoma

\section{MTTR}

\begin{tabular}{ccc}
\multirow{2}{*}{ Equipamentos } & Antes da implantação & Após da implantação \\
\cline { 2 - 3 } & Horas/ minutos & Horas/ minutos \\
\hline TM 16488 & $3: 02: 01 / 182$ & $1: 55: 42 / 115$ \\
TM 20500 & $1: 32: 00 / 92$ & $0: 54: 17 / 54$ \\
TM 18375 & $2: 15: 27 / 135$ & $1: 06: 65 / 66$ \\
Média & 136 minutos & 78 minutos \\
\hline
\end{tabular}

No equipamento TM 20500, o reparo médio foi de 01 hora e 32 minutos de reparo. No equipamento TM 18375, foi de 02 horas e 15 minutos. Por fim, no equipamento TM 16488, foi de 03 horas e 02 minutos. Estas médias foram elevadas, devido em parte a quebras de peças que a empresa não dispunha em estoque e em parte devido à necessidade de mobilização da manutenção central, mesmo em problemas simples. Com estes solucionados de imediato pelos próprios operadores e a manutenção de peças em estoque local, houve sensível redução de quebras. No período, o tempo médio até o reparo caiu em mais de $40 \%$.

A Tabela 3 apresenta dados comparados entre as máquinas escolhidas como piloto para o custo total de manutenção em $\mathrm{R} \$$.

Tabela 3 - Custos de manutenção antes e após a implantação da manutenção autônoma Custos de Manutenção

\begin{tabular}{ccc}
\hline \multirow{2}{*}{ Equipamentos } & Antes da implantação & Após a implantação \\
\cline { 2 - 3 } & $\mathrm{R} \$$ & $\mathrm{R} \$$ \\
\hline TM 16488 & 12.570 & 5.390 \\
TM 20500 & & \\
TM 18375 & 6.285 & 2.695 \\
\hline
\end{tabular}

Em novembro e dezembro de 2012, nos três equipamentos, os custos de manutenção mecânica e eletroeletrônica foram respectivamente de $R$ \$ 8.875 e $R$ \$ 3.695 totalizando $\mathrm{R} \$ 12.570$. Em julho e agosto de 2013 , o custo baixou para $\mathrm{R} \$$ Revista Produção Online, Florianópolis, SC, v.15, n. 4, p. 1123-1147, out./dez. 2015. 
5.390. A média mensal caiu a quase $60 \%$ do valor original. O custo de manutenção foi calculado somando-se custos de materiais e custos de mão-de-obra externa eventualmente contratada especificamente para as atividades requeridas para garantir a produção. Não foi computado um pequeno custo de antecipação de estoques. Também não foram computados os custos decorrentes de perda de produção por paradas não-programadas e custos de mão-de-obra fixa, dado que este custo não variou em função do início das atividades de manutenção autônoma. Houve, por ora, apenas um rearranjo na alocação da força de trabalho disponível na fábrica.

Em síntese, observaram-se expressivas melhorias no MTBF e no custo de manutenção e uma melhoria aceitável no MTTR. Estas melhorias foram possíveis devido às intervenções de manutenção que foram planejadas nos equipamentos no decorrer do projeto para solucionar falhas ostensivas e possíveis falhas ocultas. Houve também uma maior responsabilidade e comprometimento da equipe operacional com o estado do equipamento: antes, este comprometimento era exigido apenas do setor de manutenção central. A manutenção autônoma e a utilização de check-list foram contributivos para a identificação de problemas, que muitas vezes foram de rápida e simples solução. Outra iniciativa que contribuiu para reduzir os tempos de reparo foi a implantação de um pequeno estoque de peças de reposição, incluindo os itens de maior consumo. A busca por estes itens, em situação de emergência, era o fator que mais retardava a reposição dos equipamentos após uma quebra inesperada.

A Figura 3 apresenta os tipos e as frequências dos problemas que foram detectados pela aplicação do check-list nas três máquinas. As atividades de correção que a detecção precoce provocou evitaram possíveis paradas nãoprogramadas, ajudando a explicar a elevação do MTBF. A alocação prévia de material no almoxarifado ajuda a explicar a redução do MTTR. 
Figura 3 - Tipos de paradas detectadas pelo check-list

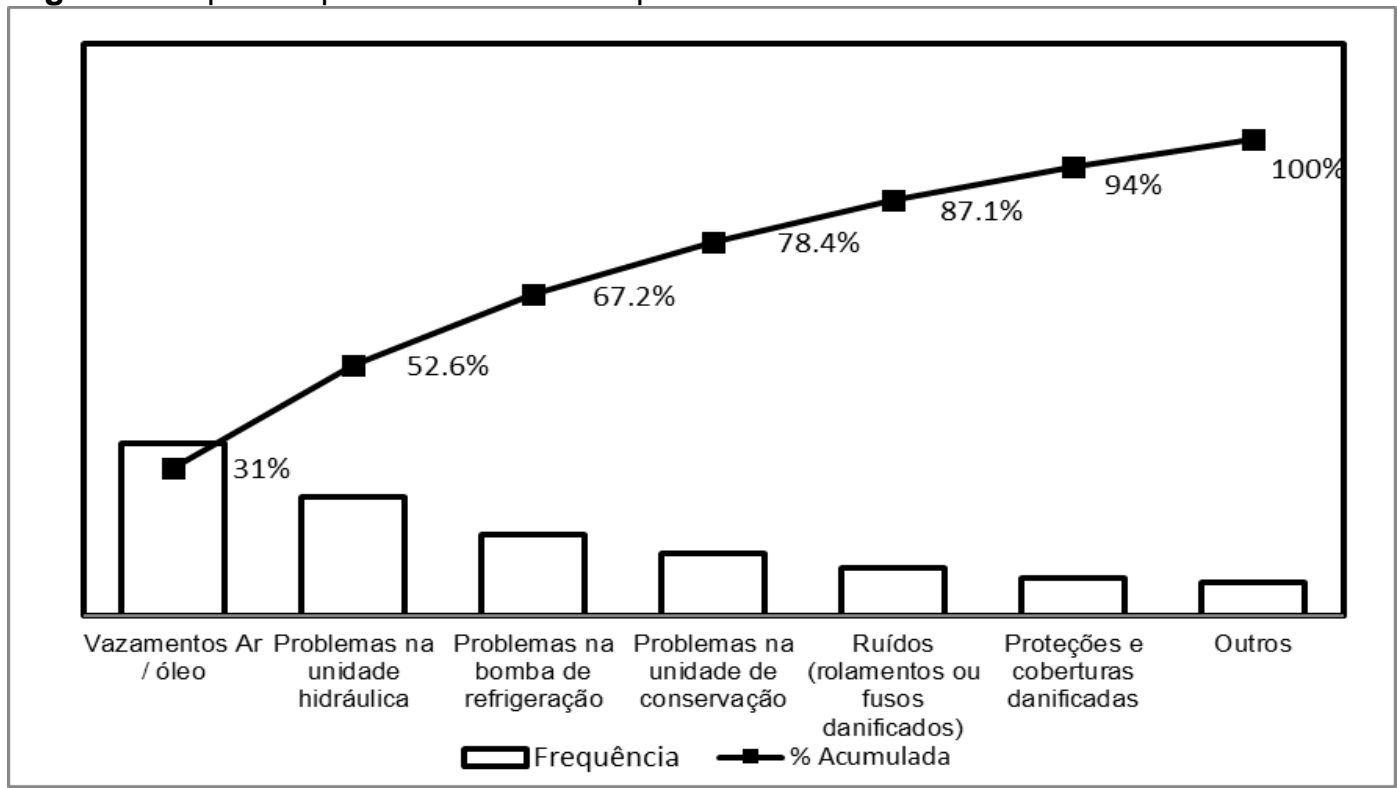

A Figura 4 apresenta a evolução da distribuição de atividades entre a equipe operacional e a manutenção central. Observa-se que, ao fim do período estudado, a equipe operacional já estava executando quase a metade das atividades de manutenção corretiva, de emergência ou de melhorias. Com isto, a manutenção central passou a usar mais recursos em manutenção planejada e preventiva, tipicamente reformas de máquinas e troca de tecnologia.

Figura 4 - Percentual de atividades conduzidas pela manutenção central e pela equipe operacional

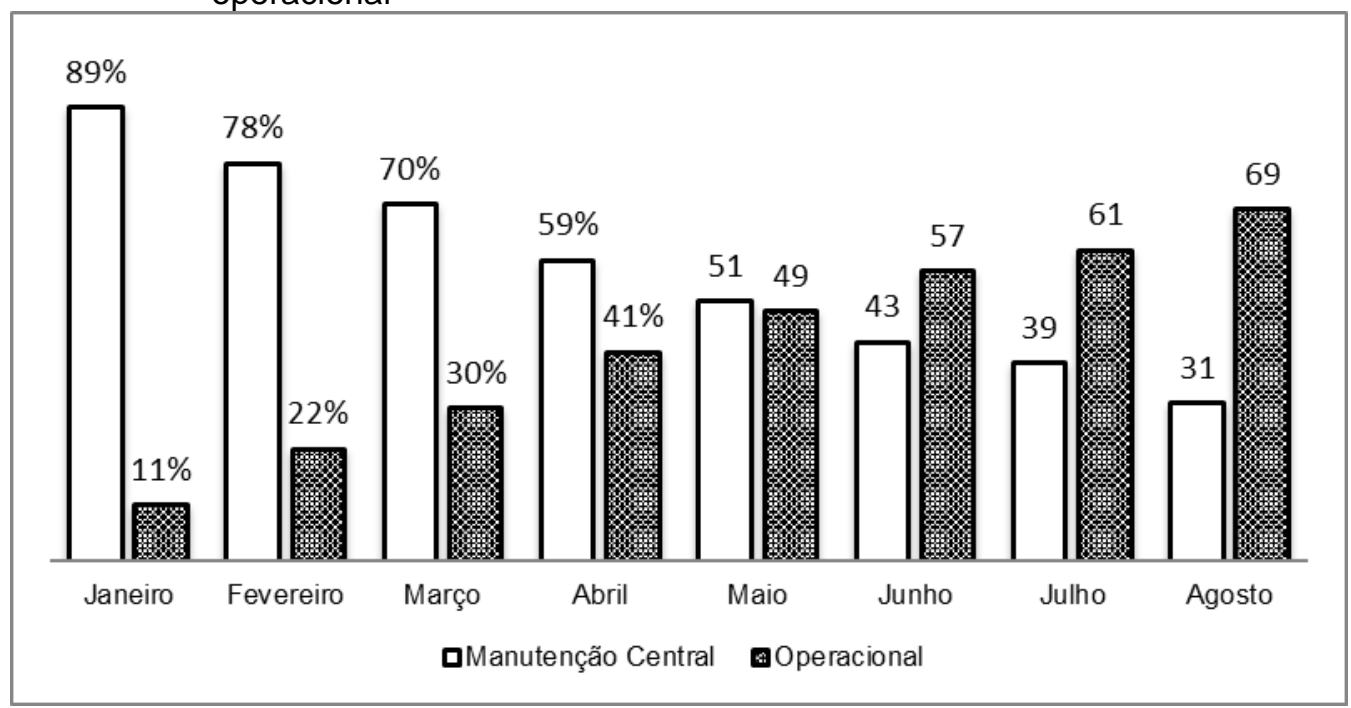

Revista Produção Online, Florianópolis, SC, v.15, n. 4, p. 1123-1147, out./dez. 2015. 
Houve alguma dificuldade de aceitação do programa de manutenção autônoma por parte dos funcionários. Porém, no decorrer do programa, estes foram se adequando ao sistema e a nova filosofia. Alguns benefícios da nova forma de trabalhar já foram percebidos por alguns dos envolvidos. Espera-se que, na sequência das atividades, o nível de conscientização das equipes de operadores e dos técnicos de manutenção sobre os benefícios potenciais da manutenção autônoma cresça junto com os resultados de campo.

Dentre os resultados observados, incluem-se alguns não-tangíveis, destacados pela equipe de implantação: uma primeira experiência de autogestão dos recursos de produção foi construída; aumento expressivo no nível de comprometimento da equipe de operadores na busca de um estado ideal de zero falha de máquina e zero defeito de produto; e surgimento de relação sinérgica positiva entre operadores e técnicos da manutenção na busca do aperfeiçoamento dos processos produtivos.

Os resultados iniciais apontaram aumento no intervalo entre as falhas $\mathrm{e}$ redução dos tempos de reparo dos equipamentos. Portanto, houve aumento na disponibilidade dos mesmos. Usando a equação (1) (SELLITTO, 2005) para cálculo da disponibilidade $A v$, verifica-se que, para as três máquinas estudadas, a mesma passou de $94,2 \%$ para $99,7 \%$.

$$
A v=\frac{M T B F}{M T B F+M T T R}
$$

O acréscimo de cerca de cinco pontos percentuais à disponibilidade de máquinas, considerando turno de 16 horas diárias e seis dias por semana para as três máquinas, acrescentou mais cerca de 20 horas mensais de máquina à produção. Este resultado confirma o que a revisão apontou: atividades de TPM e em particular de manutenção autônoma, podem aumentar a capacidade de máquina disponível para produção e eventualmente dispensar investimentos em novas máquinas. 


\section{CONSIDERAÇÕES FINAIS}

O objetivo deste artigo foi descrever um caso de aplicação de manutenção autônoma em uma área-piloto de uma empresa da indústria metal-mecânica, a empresa Alfa. O método de pesquisa foi o estudo de caso. A questão de pesquisa foi: como aplicar a manutenção autônoma em uma empresa da indústria metalmecânica?

A questão de pesquisa foi respondida introduzindo na empresa Alfa um programa de implementação de práticas de TPM, iniciando pela manutenção autônoma. Um programa de doze pontos foi estruturado e adotado como guia de implantação da manutenção autônoma. O programa iniciou por uma área-piloto, incluindo três centros de usinagem. Os principais resultados do programa-piloto foram o aumento em mais de $700 \%$ no MTBF, a redução de mais de $40 \%$ no MTTR, o consequente aumento de mais de cinco pontos percentuais na disponibilidade das três máquinas e a redução de quase $60 \%$ no custo de materiais de manutenção.

A principal conclusão que se pode extrair do estudo foi que a TPM, e em particular a manutenção autônoma, pode aumentar a eficiência da manutenção como fator estratégico e consequentemente pode aumentar a capacidade de competição da manufatura de uma empresa industrial.

Como continuidade de pesquisa, aponta-se a necessidade de observar se houve redução de defeitos nos produtos fabricados nos três centros de usinagem estudados. É de se esperar que a redução de quebras de máquinas e a eliminação de problemas crônicos originados de erros de projeto, de instalação ou de operação também tenham produzido reflexos em outros aspectos da produção, tal como a qualidade dos produtos acabados. Métricas convenientes para este aspecto são o percentual de refugos e o percentual de retrabalhos observados nas três máquinas nos períodos de estudo. Outra oportunidade de pesquisa está em acompanhar os futuros desenvolvimentos da TPM e implantações em outras áreas, de modo a gerar mais estudos de caso locais, como este, e um estudo de caso global, relatando a implantação da TPM por toda a empresa. 


\section{AGRADECIMENTOS}

Parte da pesquisa foi financiada pelo CNPq.

\section{REFERÊNCIAS}

AHMED, S.; HASSAN, M.; TAHA, Z. TPM can go beyond maintenance: excerpt from a case implementation. Journal of Quality in Maintenance Engineering, v.11, n.1, p.19-42, 2005. http://dx.doi.org/10.1108/13552510510589352

AHUJA, I. Exploring the impact of effectiveness of total productive maintenance strategies in manufacturing enterprise. International Journal of Productivity and Quality Management, v.9, n.4, p.486-501, 2012. http://dx.doi.org/10.1504/IJPQM.2012.047194

AHUJA, I.; KHAMBA, J. Strategies and success factors for overcoming challenges in TPM implementation in Indian manufacturing industry. International Journal of Quality in Maintenance Engineering, v.14, n.2, p.123-147, 2008.

http://dx.doi.org/10.1108/13552510810877647

ALCARAZ, J. Factores relacionados con el éxito del mantenimiento productivo total. Revista Facultad de Ingeniería Universidad de Antioquia, Colombia, n. 60, v.1, p.129-140, 2011.

BAMBER, C.; SHARP, J.; HIDES. M. Factors affecting successful implementation of total productive maintenance: a UK manufacturing case study perspective. Journal of Quality in Maintenance Engineering, v.5, n.3, p.162-181, 1999.

http://dx.doi.org/10.1108/13552519910282601

BESSANT, J. Developing continuous improvement capability. International Journal of Innovation Management, v.2, n.4, p.409-429, 1999.

http://dx.doi.org/10.1142/S1363919698000183

BESSANT, J.; CAFFYN, S. High involvement innovation through continuous improvement. International Journal of Techology and Management, v.14, n.1, p.7-28, 1997. http://dx.doi.org/10.1504/IJTM.1997.001705

BESSANT, J.; CAFFYN, S.; GALLAGHER, M. An evolutionary model of continuous improvement behavior. Technovation, v. 21, n.2, p.67-77, 2001.

http://dx.doi.org/10.1016/S0166-4972(00)00023-7

BESSANT, J.; CAFFYN, S.; GILBERT, J.; HARDING, R.; WEBB, S. Rediscovering continuous improvement. Technovation, v14, n.1, p.17-29, 1994.

BHUIYAN, N.; BAGHEL, A. An overview of continuous improvement: from the past to the present. Management Decision, v. 43, n. 5, p.761-771, 2005.

http://dx.doi.org/10.1108/00251740510597761

BOER, H.; GERTSEN, F. The continuous improvement to continuous innovation: a retro perspective. International Journal Technology Management, v. 26, n. 8, p.805-827, 2003. http://dx.doi.org/10.1504/IJTM.2003.003391 
CHAN, F.; LAU, H.; IP, R.; CHAN, H.; KONG, S. Implementation of total productive maintenance: a case study. International Journal of Production Economics, v. 95, n.1, p.71-94, 2005. http://dx.doi.org/10.1016/j.ijpe.2003.10.021

DOGRA, M.; SHARMA, V.; SACHDEVA, A.; DUREJA, J. TPM - a key strategy for productivity improvement in process industry. Journal of Engineering Science and Technology, v.6, n.1, p.1-16, 2011.

GOMES, M.; LIMA, C.; SILVA I. Implantação da Lubrificação Autônoma como Ferramenta Essencial do TPM: Uma Abordagem Prática. ENCONTRO NACIONAL DE ENGENHARIA DE PRODUÇÃO. Anais ... Bento Gonçalves: ABEPRO, 2012.

GRAISA, M.; AL-HABAIBEH, A. An investigation into current production challenges facing the Libyan cement industry and the need for innovative total productive maintenance (TPM) strategy. Journal of Manufacturing Technology Management, v. 22, n.4, p. 541-558, 2011. http://dx.doi.org/10.1108/17410381111126445

JHA, S.; NOORI, H.; MICHELA, J. The dynamics of continuous improvement. International Journal of Quality Science, v.1, n.1, p.19-47, 1996.

http://dx.doi.org/10.1108/13598539610117975

KARDEC, A.; NASCIF, J. Manutenção função estratégica. Rio de Janeiro: Qualitymark editora, 1998.

$\mathrm{KOCH}, \mathrm{A}$. Discover the hidden machine: OEE for production team. Amsterdam: FullFact $\mathrm{BV}, 2007$.

LAZIM, H.; RAMAYAH, T.; AHMAD, N. Total productive maintenance and performance: A Malaysian SME experience. International Review of Business Research Paper, v.4, n.4, p. 237-250, 2008.

LEÃO, S. Aplicação da troca rápida de ferramentas (TRF) em intervenções de manutenção preventiva. Produção Online, v.9, n.1, p.1-26, 2009.

http://dx.doi.org/10.14488/1676-1901.v9i1.205

LJUNGBERG, O. Measurement of Overall Equipment effectiveness as a basis for TPM activities. International Journal of Operations and Production Management, v.18, n.5, p.495-507, 1998. http://dx.doi.org/10.1108/01443579810206334

MCKONE, K.; SCHROEDER, R.; CUA, K. The impact of total productive maintenance practices on manufacturing performance. Journal of Operations Management, v.19, n.1, p. 39-58, 2001. http://dx.doi.org/10.1016/S0272-6963(00)00030-9

MONTEIRO, A.; MONTEIRO, D.; MOTTA, D.; SILVA, D. Proposta de aumento de eficiência fabril por meio da manutenção produtiva total em uma empresa fabricante de embalagem de alumínio. ENCONTRO NACIONAL DE ENGENHARIA DE PRODUÇÃO. Anais ... Bento Gonçalves: ABEPRO, 2012.

MUTHIAH, K.; HUANG, S.; MAHADEVAN, S. Automating factory performance diagnostics using overall throughput effectiveness (OTE) metric, International Journal of Advanced Manufacturing Technology, v. 36, n.7/8, p. 811-824, 2008.

http://dx.doi.org/10.1007/s00170-006-0891-x 
NAKAJIMA, S. Introdução ao TPM - Total Productive Maintenance. São Paulo: IMC Internacional Sistemas Educativos Ltda., 1989.

NAKAJIMA, S. Introduction to Total Productive Maintenance (TPM). Cambridge: Productivity Press, 1988.

NAKAMURA, T. Total productive maintenance. New York: John Wiley \& Sons, 2007.

NAKAZATO, K. Conceitos básicos do TPM parte II. Japão: JIPM, Japan Institute of Plant Maintenance. 2000.

NAKAZATO, K. Manual de implementação do TPM. Japão: JIPM, Japan Institute of Plant Maintenance. 1999.

PACHECO, D.; ANTUNES JÚNIOR, J.; LACERDA, D.; GOLDMEYER, D.; GILSA, C.; Modelo de gerenciamento da capacidade produtiva: integrando teoria das restrições e o índice de rendimento operacional global (IROG). Produção Online, v.12, n.3, p. 806-826, 2012. http://dx.doi.org/10.14488/1676-1901.v12i3.981

PETTER, R.; RESENDE, L.; SELIG, P.; VAZ, C. Produção limpa, produção mais limpa, produção enxuta, 5s e manutenção autônoma: uma proposta metodológica de implantação conjunta. CONGRESSO NACIONAL DE EXCELÊNCIA EM GESTÃO. Anais.... Rio de Janeiro: UFF, 2011.

RAJPUT, H.; JAYASWAL, P. A Total Productive maintenance (tpm) approach to improve overall equipment efficiency. International Journal of Modern Engineering Research, v.2, n.6, p.4383-4386, 2012.

RAPOSO, C. Overall equipment effectiveness: aplicação em uma empresa do setor de bebidas do pólo industrial de Manaus. Produção Online, v.11, n.3, p.648-667, 2011. http://dx.doi.org/10.14488/1676-1901.v11i3.529

SALVENDY, G. Handbook of industrial engineering. New York: John Wiley \& Sons, 1992.

SELLITTO, M. Inteligência artificial: uma aplicação em uma indústria de processo contínuo.

Gestão \& Produção, v.9, n.3, p.363-376, 2002.

http://dx.doi.org/10.1590/S0104-530X2002000300010

SELLITTO, M. Formulação estratégica da manutenção industrial com base a confiabilidade dos equipamentos, Produção, v.15, n. 1, p. 44-59, 2005. http://dx.doi.org/10.1590/S0104-530X2002000300010

SELLITTO, M.; BORCHARDT, M.; ARAÚJO, D. Manutenção centrada em confiabilidade: aplicando uma abordagem quantitativa. ENCONTRO NACIONAL DE ENGENHARIA DE PRODUÇÃO. Anais... Curitiba: ABEPRO, 2002. http://dx.doi.org/10.1590/S0103-65132005000100005

SHIROSE, K. TPM for Workshop Leaders. Portland: Productivity Press, 1992.

SHIROSE, K; BODEK, N. TPM for workshop leaders. New York: Productivity Press, 1992.

SINGH, M.; SACHDEVA, A.; BHARDWAJ, A. An interpretive structural modelling approach for analysing barriers in total productive maintenance implementation. International Journal of Industrial and Systems Engineering, v.16, n.4, p. 433-450, 2014. 
SINGH, R.; GOHIL, A.; SHAH, D.; DESAI, S. Total Productive Maintenance (TPM) implementation in a machine shop: A case study. Procedia Engineering, v.51, n.1, 592-599, 2013. http://dx.doi.org/10.1016/i.proeng.2013.01.084

SIVAKUMAR, D. Application of total productive maintenance to reduce non-stick on pad problem in IC packaging. International Journal of Engineering and Science, v.3, n.1, p.14-32, 2012.

SOUZA, J. A Manutenção produtiva total na indústria extrativa mineral: a metodologia TPM como suporte de mudanças. Dissertação de Mestrado em Engenharia de Produção. Florianópolis: Universidade Federal de Santa Catarina, 2001.

SUZUKI, T. TPM in process industries. Portland: Productivity Press, 1994

TAKAHASHI, Y.; OSADA, T. Manutenção produtiva total. São Paulo: IMAM, 2000.

TAKAHASHI, Y.; OSADA, T. TPM/MPT: manutenção produtiva total. São Paulo: IMAM, 1993.

TAVARES, L. Administração moderna da manutenção. Rio de Janeiro: Novo Polo, 1999.

TONDATO, R. Manutenção produtiva total: estudo de caso na indústria gráfica.

Dissertação de Mestrado em Engenharia de Produção. Porto Alegre: Universidade Federal do Rio Grande do Sul, 2004.

TSANG, A.; CHAN, P. TPM implementation in China: a case study. International Journal of Quality \& Reliability Management, v.17, n. 2, p.144-157, 2000.

http://dx.doi.org/10.1108/02656710010304555

TSUCHIYA, S. Quality maintenance: zero defects through equipment management. Cambridge: Productivity Press, 1992.

VENKATESH, J. Introduction to total productive maintenance (TPM). The Plant Maintenance resource Center, 2003. Disponível em:

<www.plantmaintenence.com/articles/tpm_intro.shtml>. Acesso em: 09 abr. 2013.

WIREMAN, T. Total Productive Maintenance: an American Approach. New York: Industrial Press Inc., 2004.

WYREBSKI, J. Manutenção produtiva total: um modelo adaptado. Dissertação de Mestrado em Engenharia de Produção. Florianópolis: Universidade Federal de Santa Catarina, 1997.

YAMAGUCHI, C. TPM - Manutenção produtiva total. São João Del Rei : ICAP - Instituto de Consultoria e Aperfeiçoamento Profissional Del-Rei, 2005.

YOSHICAZEM, O. Manutenção produtiva total. São Paulo: IMAN, 2002.

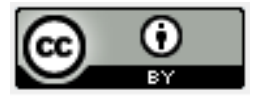

Artigo recebido em 01/07/2013 e aceito para publicação em 13/10/2014

DOI: http://dx.doi.org/ 10.14488/1676-1901.v15i4.1632 